\section{Wegener's granulomatosis in a young patient preceded by localized cutaneous manifestations}

\author{
Jesper Smit, Jakob Lykke Poulsen, ${ }^{2}$ \\ Jakob Solling, ${ }^{3}$ Carsten Sauer Mikkelsen ${ }^{4}$ \\ 'Department of Infectious Diseases, \\ Aalborg Hospital, Aarhus University \\ Hospital, Aalborg; 'Department of \\ Internal Medicine, Lillebaelt Hospital, \\ Vejle; ${ }^{3}$ Department of Nephrology, \\ Aalborg Hospital, Aarhus University \\ Hospital, Aalborg; ${ }^{4}$ Private Practice, \\ Bredgade 13, Broenderslev, Denmark
}

\begin{abstract}
Wegener's granulomatosis (WG) is a rare, systemic vasculitis involving multiple organs. The clinical presentation is highly diverse, and there is considerable risk of mortality if diagnosis and treatment are delayed. We present a case illustrating that patients with WG may initially present with localized cutaneous symptoms and signs.
\end{abstract}

\section{Introduction}

Wegener's granulomatosis (WG) is an uncommon necrotizing vasculitis that most commonly affects the upper airways, lungs and kidneys, but can involve any other organ. ${ }^{1,2}$ The disease presents with varying symptoms and signs and early recognition and initiation of adequate immunomodulatory therapy are essential in limiting the potentially life-threatening aspects of the disorder. ${ }^{1,3-4}$ We report a case illustrating that WG may present with localized, cutaneous manifestations preceding systemic disease.

\section{Case Report}

An 18-year old Caucasian man presented with a painful rash on the truncus that had developed over the course of a few weeks. The patient was known to have type-1 diabetes mellitus since the age of 8 , but was otherwise healthy and reported no recent travel activity or antecedent trauma to the affected area. Objective examination of the skin revealed inflamed cystic and nodular lesions confined to the chest and left shoulder, but there was no significant suppuration or other apparent signs of infection. The condition resembled a case of severe acne, and initial histopathological examination of a skin biopsy supported this preliminary clinical diagnosis.

A few weeks later the patient developed a sore throat and signs of upper respiratory tract infection including cough, dyspnoea and mild chest pain. The skin changes remained limited to the chest and shoulder but had clearly progressed and now appeared as deep vasculitic ulcerations, including multiple elements more than $0.5 \mathrm{~cm}$ deep (Figure 1). These findings and symptoms were accompanied by several weeks of fever (up to $39.6^{\circ} \mathrm{C}$ ), malaise and substantial unilateral facial pain, and the patient was admitted to a department of nephrology for further investigation and treatment.

The blood leukocyte and thrombocyte counts were normal as were liver and renal function tests, but C-reactive protein (CRP) was 300 $\mathrm{mg} / \mathrm{L}$ (normal range, less than $10 \mathrm{mg} / \mathrm{L}$ ) and anti-neutrophil cytoplasmic antibodies including specific identification of proteinase 3 (cANCA/PR3-ANCA) was $223 \mathrm{kU} / \mathrm{L}$ (normal range, less than $7 \mathrm{kU} / \mathrm{L}$ ). During hospitalization the patient's kidney function was continually assessed and monitored, but levels of creatinine and urea remained within normal ranges and examination of urine including microbiological analysis revealed no abnormalities.

Chest X-ray examination was performed and several lung infiltrates with caverns were noted. A subsequent CT guided lung biopsy demonstrated no sign of infection, but significant inflammation of the tissue was observed, and a diagnosis of WG with concomitant mononeuritis multiplex involving the trigeminal nerve was established.

The patient received systemic methylprednisolone and cyclofosfamide pulse therapy, which resulted in prompt improvement of the clinical condition and skin lesions as well as a decrease in the CRP and ANCA titers. Shortly after, however, the patient developed fever and renewed elevation of the c-ANCA/PR3-ANCA, and treatment was supplemented with rituximab leading to immediate resolution of symptoms and no residual pulmonary or cutaneous sequelae on recent follow-up.

\section{Discussion}

Our patient initially presented with a localized rash on the truncus, but his condition was insidiously complicated by malaise, fever and progression of the cutaneous lesions, and ultimately a diagnosis of systemic WG was established. The etiology of the disease remains unknown, however, several studies suggest that infectious antigens and especially $S$.
Correspondence: Jesper Smit, Department of Infectious Diseases, Aalborg Hospital, Aarhus University Hospital, Mølleparkvej 4, P.0. Box 365, DK-9000 Aalborg, Denmark.

Tel: +45.26241332. E-mail: jesm@rn.dk

Key words: Wegener's granulomatosis, cutaneous findings, case report, vasculitis, diabetes.

Contributions: JS, JLP, JS, CSM, manuscript writing and text reviewing.

Conflict of interest: the authors have no conflict of interest.

The manuscript has not been published and is not being considered for publication elsewhere.

Received for publication: 14 September 2011. Accepted for publication: 22 September 2011.

This work is licensed under a Creative Commons Attribution NonCommercial 3.0 License (CC BYNC 3.0).

(C) Copyright J. Smit et al., 2011

Licensee PAGEPress, Italy

Dermatology Reports 2011; 3:e44

doi:10.4081/dr.2011.e44

aureus may contribute to the pathogenesis of vasculitis in susceptible hosts. ${ }^{1,5}$

The clinical presentation of WG is complex and found to be dependent on the number of organs affected and the duration of the disease. As in our case, the clinical course of WG is characterized by an initial or localized phase which may affect any organ followed by a generalized or systemic phase in approximately $80 \%$ of cases. ${ }^{1,3}$ However, in most patients specific cutaneous findings develop concurrently or after the onset of systemic involvement and affect the head or extremities, ${ }^{5}$ but in our case they were confined to the truncus and preceded systemic symptoms and signs, which has only seldomly been described. ${ }^{6}$

In WG specific cutaneous lesions are seen in approximately $15 \%$ of cases, ${ }^{5,6}$ and are usually associated with renal disease and musculoskeletal affection, but this was not noted in our patient. Patients may develop more than one type of cutaneous lesion and these may change over time or with treatment, but several studies have identified palpable purpura as the most characteristic cutaneous lesion, and nodules, papules, ulcerations and deep erythema nodusum-like subcutaneous nodules complete the clinical spectrum. ${ }^{6-8}$ Oral involvement is not uncommon in WG, ${ }^{5,7}$ but this was not observed in our patient during the course of the disease.

In this present case the level of $\mathrm{c}$-A NCA/PR3-ANCA was markedly elevated, and ANCA positivity has been found to occur in 90 $95 \%$ of cases with active WG. ${ }^{1,6}$ However, as levels correlate with disease activity and relapses 
in the absence of ANCA are rare, repeat testing and evaluation of blood biochemistry in order to follow the course of the disease is highly recommended.

As illustrated by our case the diagnosis of WG represents a challenge and is based on anamnestic information closely correlated with clinical features, pathologic findings and ANCA testing. ${ }^{9}$ Relevant differential diagnoses are numerous and include leukocytoclastic vasculitis, Henoch-Schönlein purpura, pyoderma gangrenosum, lymphoma, erythema nodosum, rheumatoid arthritis, drug reactions, and a variety of infectious conditions. ${ }^{5}$ However, like WG, these conditions often present with varying and uncharacteristic symp-

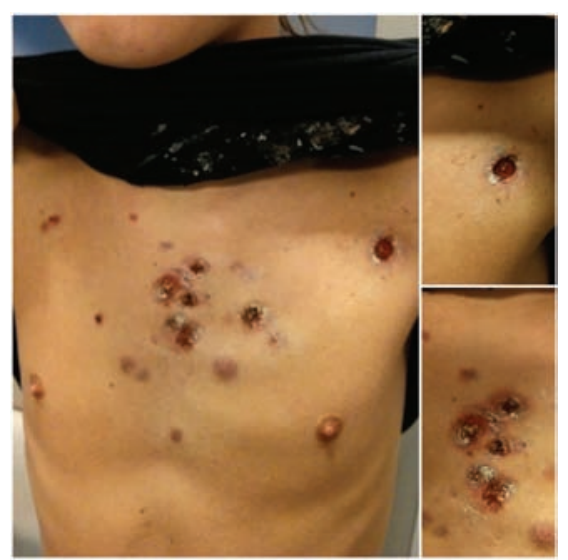

Figure 1. Cystic and nodular lesions on the chest, notice necrotic wounds with element size ranging from a few millimeters up to 2 $\mathrm{cm}$. In consistence with vasculitis the elements are inflamed and do not blanch on pressure. toms and signs and, as in our case, dermatological evaluation may provide valuable diagnostic information.

If untreated, systemic WG leads to more than $90 \%$ mortality in the first two years, hence early recognition and initiation of adequate immunomodulatory treatment are essential and associated with significantly decreased morbidity and mortality. ${ }^{1,410}$ High dose corticosteoroids and pulsed intravenous cyclofosfamide remain the mainstay of initial therapy, ${ }^{4}$ but relapse and refractory WG still represent therapeutic challenges. However, data from recent studies evaluating biological therapies, including TNF- $\alpha$ blockers (infliximab) and monoclonal antibodies (rituximab) are promising ${ }^{1,4}$ and future treatment regimes will hopefully further improve the outcome of this patient group. ${ }^{4}$

We report a case illustrating that patients with WG may initially present with localized cutaneous symptoms and signs preceding serious systemic disease. Prompt recognition of the condition and initiation of early and adequate immunomodulatory therapy is crucial in order to reduce mortality and morbidity. Dermatologists therefore need to be aware of WG as a possible differential diagnosis, especially in patients presenting with characteristic skin manifestations and accompanying systemic symptoms and signs.

\section{References}

1. Schilder AM. Wegener's Granulomatosis vasculitis and granuloma. Autoimmun Rev 2010;9:483-7.
2. Koldingsnes W, Nossent H. Epidemiology of Wegener's granulomatosis in northern Norway. Arthritis Rheum 2000;43:2481-7.

3. Rodrigues CE, Callado MR, Nobre CA, et al. Wegener's granulomatosis: prevalence of the initial clinical manifestations - report of six cases and review of the literature. Rev Bras Reumatol 2010;50:150-64.

4. Carruthers D, Sherlock J. Evidence-based management of ANCA vasculitis. Best Pract Res Clin Rheumatol 2009;23:367-78.

5. Gibson LE, Specks U, Homburger H. Clinical utility of ANCA tests for the dermatologist. Int J Dermatol 2003;42:859-69.

6. Comfere NI, Macaron NC, Gibson LE. Cutaneous manifestations of Wegener's granulomatosis: a clinicopathologic study of 17 patients and correlation to antineutrophil cytoplasmic antibody status. J Cutan Pathol 2007;34:739-47.

7. Marzano AV, Fanoni D, Berti E. Oral and cutaneous findings are valuable diagnostic aids in Wegener's granulomatosis. Eur J Intern Med 2010;21:49.

8. Barksdale SK, Hallahan CW, Kerr GS, et al. Cutaneous pathology in Wegener's granulomatosis. A clinicopathologic study of 75 biopsies in 46 patients. Am J Surg Pathol 1995;19:161-72.

9. Leavitt RY, Fauci AS, Bloch DA, et al. The American College of Rheumatology 1990 criteria for the classification of Wegener's granulomatosis. Arthritis Rheum 1990;33: 1101-7.

10. Takala JH, Kautiainen H, Leirisalo-Repo M. Survival of patients with Wegener's granulomatosis diagnosed in Finland in 1981-2000. Scand J Rheumatol 2010;39:716. 